\title{
Simplifying the design of smallsat space missions using innovative tools and platforms: beeKit and beeApp
}

\author{
Lucille Baudet (Author) \\ Customer Engagement Manager \\ Open Cosmos \\ Oxford, United Kingdom \\ lucille@open-cosmos.com
}

\author{
Daniel Sors Raurell (Co-Author) \\ Head of Customer Success \\ Open Cosmos \\ Oxford, United Kingdom \\ dani@open-cosmos.com
}

\author{
Jorge Cano Martinez (Co-Author) \\ Customer Success Engineer \\ Open Cosmos \\ Oxford, United Kingdom \\ jorge@open-cosmos.com
}

\begin{abstract}
There are three main barriers in the space sector that slow down the development of space applications, science and technology: high costs, consuming paperwork and complex technology. Open Cosmos tackles these barriers, putting satellite technology in the hands of more people than ever before. This is achieved with beeKit, a payload hardware emulator platform, and beeApp, a cloud-based mission and system simulator software. By using standardised interfaces and processes together with industry best practices, the entire development of a mission can be simplified massively reducing cost and time to orbit and opening space access to a broader and more diverse audience.
\end{abstract}

The beeApp and beeKit bundle offers a seamless transition from payload concept to payload in space. beeKit replicates the mechanical and electrical constraints of a satellite platform while still being modular enough to enable payload developers to change the configuration and physical dimensions. beeApp enables full online mission with space simulation capabilities when the payload is assembled in beeKit. The Mission and System Design (MSD) module allows users to run simulations and optimise different mission parameters based on the payload requirements. The Hardware-In-the-Loop (HIL) module interfaces with the payload through beeKit, enabling smooth interaction and testing capabilities from day one. These constitute a set of groundbreaking tools that simplify the process of sending payloads to space.

Open Cosmos supports the education community by making those tools accessible for education projects. In 2018, a team of master students from Oxford University, without any space-related background, was able to conduct a biological experiment design for a $3 \mathrm{U}$ satellite platform. For the mission analysis and simulation phase, beeApp was used to select the main mission parameters. A payload into a beeKit was then designed to host cell culture flasks to control and monitor the cell death evolution. This approach facilitates cross-industrial research, at faster development rates, in a simple way and at much lower costs, enabling all kinds of organisations to conduct experiments in space.

Open Cosmos has recently established a program allowing universities from around the world to benefit from these innovative tools for free, supporting research activities and space-related education for students worldwide. This set of tools are now being used in $\mathbf{2 0}+$ different countries, in universities not only based in Europe, but globally, including space emerging countries. The goal is to continue enabling the development of new space technologies and applications and support space education programmes across the world.

Keywords- Space Mission Design, Space Education, Mission simulation, Mission Analysis, Payload, Smallsat

\section{INTRODUCTION}

The new space environment includes the emergent private space industry, specifically related to the community of space companies working to develop low-cost access to space technologies, as well as advocates of low-cost space technology and policy. New simple space mission development with decreasing launch costs for small satellites [2] allows an increased amount of satellites being deployed into LEO in short times and at reasonable costs. This trend opens the opportunity to create new capabilities and establish new business models around small satellite constellations. With the low latency telecommunications and high-speed data transmission capacities provided, it will become possible to provide satellite services over larger areas and increase the connectivity with these areas. 
This can enhance existing programs, such as remote medical support, remote education services or support to agricultural exploitation. Furthermore, the new panorama of satellite constellations will improve the establishment of communication networks in disaster areas or environment monitoring within others.

ESA has well acknowledged this new space context, promoting what is called the Space 4.0 era. It follows the Space 1.0 era (early days of astronomy), the Space 2.0 era (space race) and the Space 3.0 (ISS) era; so the main players now are the new increased number of diverse space actors around the world, including the emergence of private companies, the active participation of academia, industry and citizens, digitalisation and global interaction. This new era is unfolding through interaction between governments, the private sector, society and politics, with promising future missions ahead including new game-changing technologies.

\section{Space Mission Design Tools}

Open Cosmos approaches the new space era making satellite technologies easier and more accessible. This is achieved with beeKit, a payload hardware emulator platform, and beeApp, a cloud-based mission and system simulator platform.

\section{A. beeApp: Space Mission Design software}

Open Cosmos has developed beeApp, a cloud-based software platform that enables full mission and system design with simulation capabilities to develop space missions. It allow users to:

- Perform mission analysis studies based on the payload characteristics that the user defines and the platform solution that Open Cosmos suggests.

- Interact with beeKit in a Hardware-In-the-Loop (HIL) environment.

beeApp includes different modules that can be used depending on mission requirements. The development page is divided into different tools such as the Mission and System Design (MSD) and the beekit Hardware-In-the-Loop (HIL) tools. The Mission and System Design (MSD) module allows the user to perform mission analysis studies by running simulations based on the mission parameters. It hosts two main different sections, the inputs section and the outputs section only visible after running a specific simulation.

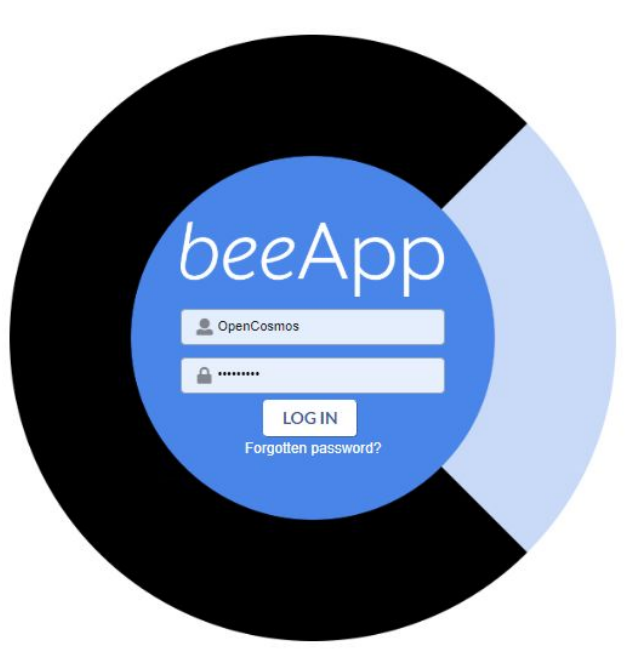

Fig. 1: beeApp login portal page
beeApp is conceived to support payload developers during all stages of space mission development including high performance parameters typically from deep space long duration missions. The second module, called Hardware-In-the-Loop (HIL), allows each user to interact with their payload and test it.

\section{B. beeKit: Satellite qualification platform}

beeApp enables full mission and systems development including simulation with hardware-in-the-loop when the payload is assembled into beekit. Users can start testing and operating their payloads from day one and in a similar way as they will do it during the operations phase in space. It also simulates the behaviour of the platform configured by the user emulating its performance and constraints from the orbital parameters and subsystems selected. Once the payload is flight ready, it is integrated and tested into the chosen platform configuration and placed into the selected orbit via Open Cosmos approved launch providers, minimising time and cost. Once in orbit, the user can control the payload from beeApp in the same way as during development phases while beeApp takes care of limiting the operational boundaries. These boundaries are set by the system configuration, mission parameters and the ground segment providers partnering with Open Cosmos with the objective to minimise downtime and increase reliability. 


\section{SUPPORTING SPACE EDUCATION}
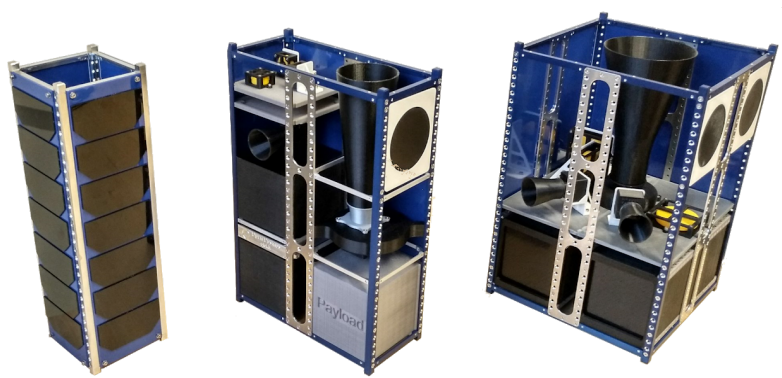

Fig, 2: 3U, $6 U \&$ \& $12 U$ beeKit platform configuration

The HIL module allows the user to deploy software and perform functional tests of its payload once physically integrated into the beeKit satellite qualification platform.

It contains the following features:

- File Management: the user can upload scripts/code to beeKit and execute commands

- MSD Interaction: run scenarios on beeKit simulated through MSD to validate payload performance

- Automatic Test Reports: users can export beeKit sensors readings \& subsystems performance.

- Operations interface: the user develops and tests the interface of the payload with beeKit the same way it would be done at a later point once in orbit.

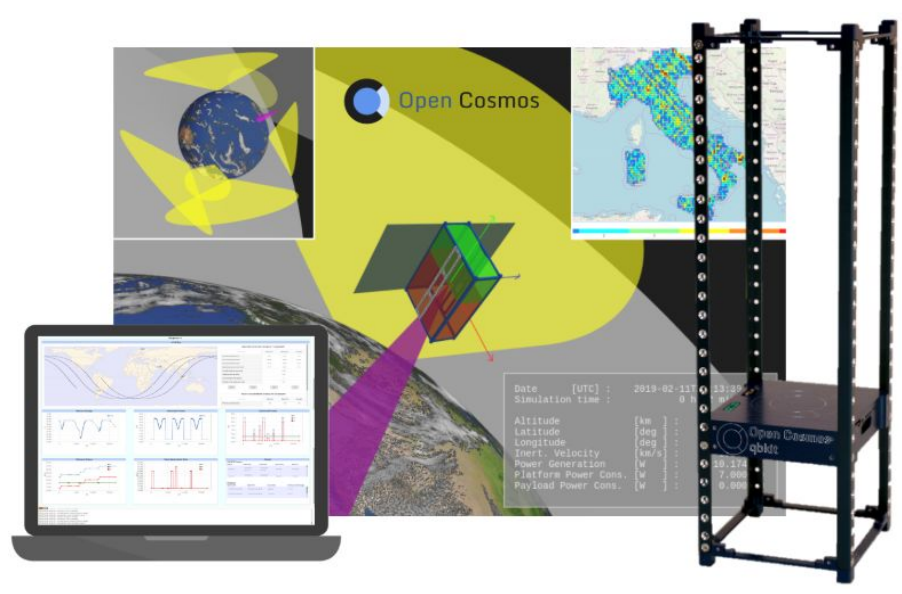

Fig.3: Simulation scenario with Hardware-in-the-loop module

\section{A. University Partnership Program}

In line with Open Cosmos' ongoing efforts to democratise access to space and to enable using space as a tool, we are supporting and empowering the student community at University level with our tools to develop space technologies and applications.

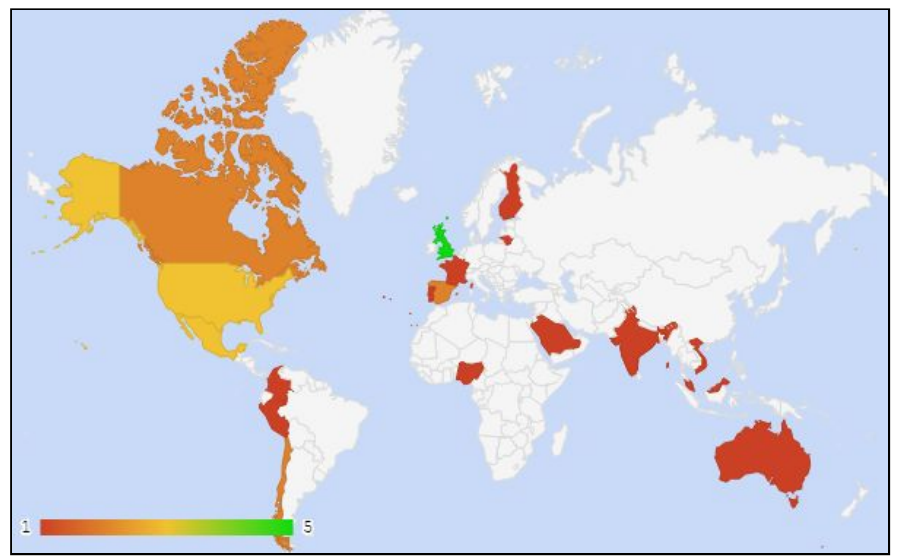

Fig.4: Open Cosmos' university partnerships worldwide

Open Cosmos has recently established a program allowing universities from around the world to benefit from these innovative tools for free, supporting research activities and space-related education for students across the world. This set of tools is now being used in $20+$ different countries, in universities not only based in Europe, but globally, including space emerging countries, enabling the development of new technologies in space education programmes worldwide.

Open Cosmos supports the young community by making those tools accessible for education projects and emerging country activities by making use of a set of programs that allows the use of the Open Cosmos tools for free. The goal is to continue enabling the development of new space technologies and applications and support space education. In 2018, a team of master students from Oxford University, without any space-related background, was able to conduct a biological mission and experiment design and prototype for a $3 \mathrm{U}$ satellite platform. For the mission analysis and simulation phase, beeApp was used to select the main mission parameters. A payload into a beeKit was then designed to host cell culture flasks to control and detect any events and to monitor the cell death evolution. The outcomes were presented during the last International Astronautical Congress held in Bremen [1], proving that this new approach of developing smallsat space mission facilitates cross-industrial research, in 
a faster, simpler and cheaper way that enables any kind of organisation to conduct experiments in space.

The company is currently supervising and supporting student projects, aiming at developing new technologies to be integrated within Open Cosmos' satellite qualification platforms (beeKits), in the UK with the University of Cranfield and France with Ecole Polytechnique, within others.

The different topics selected are enabling students to develop small satellite payloads and subsystems, being involved in a hands-on project that aims to design and build a functional demo unit of a payload (experiment, sensor, etc.) or a satellite subsystem, with its respective documentation: theoretical background, configuration instructions, list of components, assembly process, operations, etc. allowing them to acquire knowledge in many different areas.

A team from Cranfield University is focusing on the development of a proof of concept equipment of a laser communication payload capable of sending basic information that could be suitable for inter-satellite communications. The payload should be able to send and receive information to and from other payload units, so the designed equipment can operate as a transmitter and receiver. Another team of students has been working on the development of equipment to monitor biological payloads in microgravity.

Open Cosmos has also initiated a project with Ecole Polytechnique based in Paris. The team of Master students from different backgrounds in physics, mathematics will work towards putting together an ADCS demo system based on reaction wheels, to improve attitude control. Once the demonstration payload is developed, it will be integrated into the satellite platform to conduct qualification tests. This will provide a great insight to the students on key small satellite technologies and processes to qualify a new system.

Universities developing payloads or technologies are also eligible to receive for free Open Cosmos' satellite platforms for testing and integration. A university in the United States is currently working on a prototype to develop a CubeSat hyperspectral, pushbroom imager; with potentially 4 more imaging systems in 2019 that would include an imager for detecting bioluminescence from space and a thermal imager. The university is using beeApp software to simulate in-orbit operations and will be using later on beeKit platform to integrate and start testing their imager.

In the vision of supporting space technologies and applications, Open Cosmos is providing credentials to access beeApp software to any interested university. The beeKit hardware emulator can be shipped to any university premises currently developing a payload, sub-systems or technology and willing to integrate a system into a satellite platform and conduct functional and environmental tests.

\section{B. Open Cosmos outreach activities and education resources}

\section{a. Space Mission Design hands-on Workshop}

The purpose of this workshop is to provide an introduction to space mission design and planning using Open Cosmos' beeApp software platform. The curriculum of the workshop will cater to students, teachers and professionals, making use of the Mission and System Design (MSD) tool of beeApp.

This workshop serves as an introduction to the key concepts of space mission design, to learn some of the theoretical concepts relevant to satellite missions, and have the ability to apply these concepts via several hands-on exercises, using online space mission design software.

\section{b. Build, integrate and test your payload into a small satellite Workshop}

Following the Space Mission Design workshop introducing the key steps of mission design and planning, students are given the opportunity to build, integrate and test payloads into real satellite qualification platforms using beeKits.

The workshop is also focusing on the main aspects of payload design and requirements, followed by hands-on exercises allowing students to assemble either an Optical camera, IoT or biological demo payload to be integrated into OpenCosmos' beeKit platforms, and run first functional tests using the beeApp Hardware-in-the-loop module.

These two workshops have been taking place successfully in different universities around the world, receiving high appreciation and a very positive feedback.

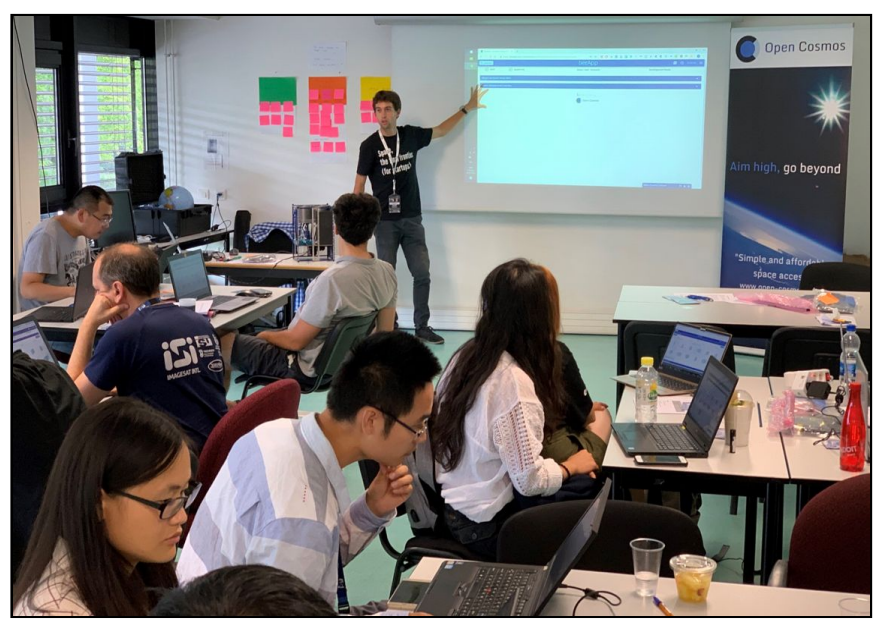

Fig.5: Open Cosmos Workshop at the International Space University SSP19 


\section{The Open Cosmos Academy}

The Open Cosmos Academy is an initiative started by Open Cosmos with the objective of promoting the development of all kinds of space technologies and applications. It consists of the use of an online open source repositories (https://gitlab.com/OC Academy) along with the corresponding documentation publicly available and open to contributions.

Thanks to the University Partnership Program, along with the hands-on Workshops, Open Cosmos is making efforts to support universities, helping to establish the basis for the next generation of space engineers and scientists that will benefit from using space as a tool. The development and results of new experiments, instruments and sensors can be documented and publicly shared through the Open Cosmos Academy, helping to distribute and expand the knowledge of space related projects across the international community.

\section{Space Emerging Countries}

Removing the main barriers from the space sector allows more players and countries to enter in the space race. Euroconconsult has identified 20 New Countries that will Invest in Space Programs by 2025, with around 130 satellites forecast to be launched by emerging space countries in the next 10 years [3]. The small satellites technology offers a good opportunity for emerging countries to get involved in space-related activities and build capability and infrastructure.

Universities are playing an important and particular role in those countries, as space emerging programs are using education and academic institutions to support the development of space capability. Satellite projects are used as education tools to drive and push the development of first-generation satellite activities, whilst educating students and engineers from the home country [4].

The Space Mission Design tools developed by Open Cosmos give support to space emerging programs, allowing access to a set of tools and resources to education. Our university partnership program along with our workshops materials is enabling the support to human resources and training from regions worldwide. Our beeApp software represents a first and simplified step toward understanding how to build and design a space mission, while our hardware emulator platform drives the development of their own capacity, from payload concept to payload in space.

The beeApp and beeKit bundle offers a simplified process to develop technologies and payload, helping the fulfillment of government priority needs for the country, while getting valuable data to solve countries issues such as land \& water management, agriculture, disaster management, etc. Main objective of those countries is to demonstrate the government their capacity to build satellite \& infrastructure, showing that it can be delivered to orbit with limited resources by individuals from the country [4]. Among 21 countries currently using beeApp, around half of them are part of emerging space countries.

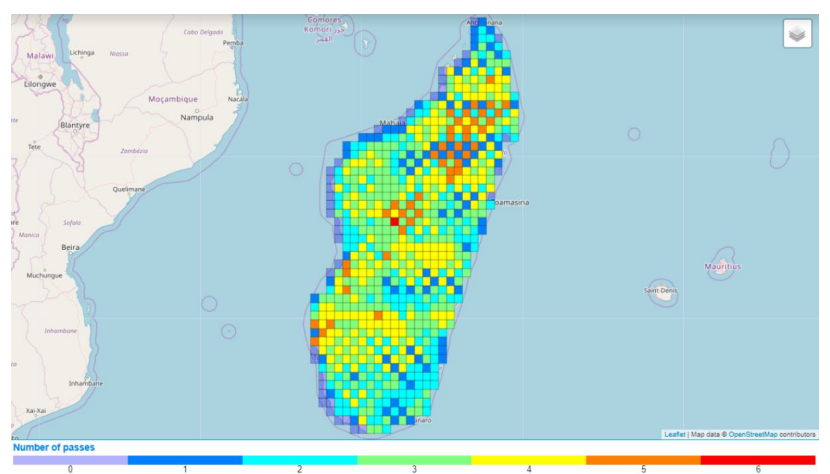

Fig. 6: Madagascar's heatmap and coverage using beeApp

\section{CONCLUSION}

Open Cosmos' set of tools included in beeKit and beeApp platforms together with the education resources developed, simplify the development of all kinds of space applications, experiments and technologies that can now be sent to space in a much faster way and at much lower costs. There have been lots of efforts done during the last 4 years in making complex technologies and processes much simpler so researchers coming from different industries can benefit from the space environment and from being in low Earth orbit. This simplified approach enables a broader and more diverse audience to access space qualified hardware and software tools to conduct experiments in space. This set of tools are already having a high positive impact in universities around the world, supporting research and educating the next generation of engineers and scientists that will contribute to improving the world we live in using space as a tool.

\section{REFERENCES}

[1] H Nazri, A. Tsai, M. Karami, The effect of microgravity and cosmic rays on immortalised human cell lines in a suspension culture condition on a nanosatellite platform. IAC-18-A2.7.10.x45177.

[2] Chin J., Coelho R., Foley J., et al, Basic Concepts and Processes for First-Time CubeSat Developers, NASA CubeSat Launch Initiative, October 2017.

[3] Euroconsult, Trends \& Prospects for Emerging Space Programs, edition 2016

[4] Polansky J. L., Cho M., Kyutech's Model for Space Engineering Capacity Building in Emerging Countries, March 31, 2016 